\title{
Maxillofacial Radiology 186
}

SADJ November 2020, Vol. 75 No. 10 p583

\section{CJ Nortjé}

Below are pictures of two cases of infection of the jaws that could be life-threatening within a few days due to spread of bacteria into perioral fascial spaces. Discuss the clinical and radiological features and what is your provisional diagnosis?

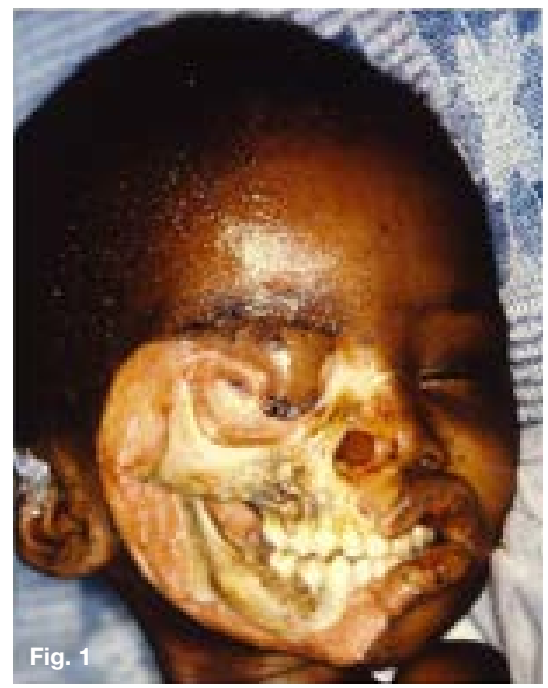

\section{INTERPRETATION}

Figure 1, is an example of an overwhelming oral infection, extending on to and destroying part of the face, and fatal if inadequately treated. It has been described as a 'severe scourge to mankind' as children are mainly affected. It also known as Cancrum Oris or Noma. It is a rapidly spreading gangrene of the oral and facial tissues that occurs usually in debilitated or nutritionally deficient persons. It occurs chiefly in persons that are undernourished or debilitated from infections such as diphtheria, dysentery, measles, pneumonia, scarlet fever, syphilis, tuberculosis and blood dyscrasias, including anemia. Thus Noma may be considered a secondary complication of a systemic disease rather than a primary disease. Noma appears to originate as a specific infection by Vincent's organisms, an acute necrotizing gigivostomatitis, which is soon complicated by secondary invasion of many other microbial forms, including streptococci, staphylococci and diphtheria bacilli. Figure 1 also shows gross destruction and sloughing of the facial tissues as a result of spread of the infection from the maxillary and mandibular gingivae. Noma was common among starving prisoners in Nazi concentration camps, particularly children in the 1940s and invariably fatal. Later, attention was drawn to the disease in Africa in the 1960's. Otherwise, noma has virtually disappeared from developed countries, and for decades the disease has been largely ignored. Figure's 2 \& 3 is a patient who presented after extraction of the 26 and 27 with proptosis and orbital cellulitis of the left eye. Axial CT reveals a large soft tissue mass extending into the ethmoid sinus, maxillary sinus and orbit

Christoffel J Nortjé: $B C h D, P h D, A B O M R, D S c$. Faculty of Dentistry, University of the Western Cape.

ORCID Number: 0000-0002-9717-5514

Email: cnortje@uwc.ac.za

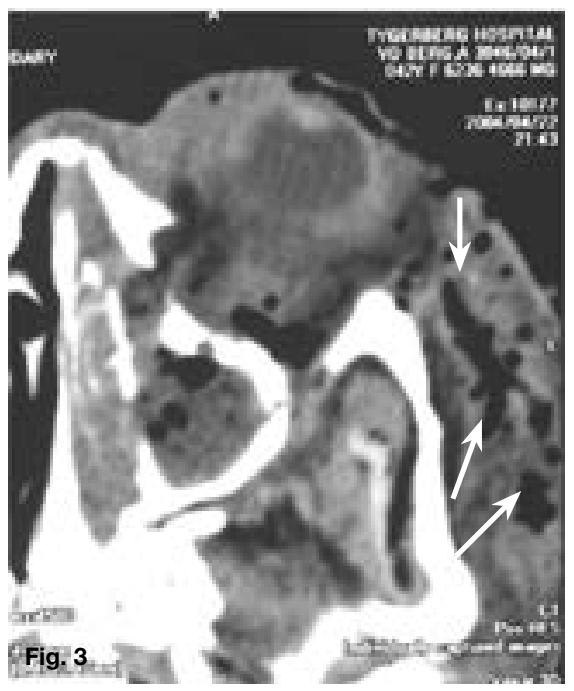

and various collections of gas (arrows) is also discernible. A diagnosis of necrotizing fasciitis (NF) of the orbit was made which is a rare and deadly condition that requires prompt surgical and medical management to decrease morbidity and mortality. The term necrotizing fasciitis was coined by Wilson in the 1950s to describe necrosis of the fascia and subcutaneous tissue with relative sparing of the underlying muscle. Necrotizing fasciitis is characterized by rapid destruction of tissue, systemic toxicity, and, if not treated aggressively, gross morbidity and mortality. Early diagnosis and aggressive surgical treatment reduces risk; however, it is often difficult to diagnose Necrotic fasciitis, and sometimes patients are treated for simple cellulitis until they rapidly deteriorate. Necrotizing fasciitis is a rapidly progressive disease of the subcutaneous tissue that carries a high mortality within hours to days without prompt medical and surgical interventions. Fasciitis of the orbit is particularly concerning because of lasting morbidity of the eye even after successful treatment of the fulminant systemic toxicity. The causative organism of NF is usually polymicrobial but streptococci and enterococci are the most common isolates. The major determinant of survival is the time from admission to the operating room for debridement. The patient's left eye had to be removed. Without treatment NF is a rapidly progressive condition and can result in significant morbidity. Management of this condition includes both medical treatment and surgical debridement. Early intervention is of vital importance to save the patient's life as septicaemia often leads to death within 24-96 hours.

\section{References}

1. Cawson RA, Odell EW. Essentials of Oral Pathology and Oral Medicine, $7^{\text {th }}$ edition. Churchill, Livingstone. 2002; 90-101.

2. Wilson B. Necrotising fasciitis. Am Surg. 1952; 18(4):416-31. 